\title{
In-Situ Liquid Transmission Electron Microscopy (TEM) for the analysis of Metal Organic Frameworks (MOFs)
}

Joseph P. Patterson ${ }^{1}$, Patricia Abellan Baeza ${ }^{2}$, Michael Denny Jr. ${ }^{1}$, Seth Cohen ${ }^{1}$, Chiwoo Park ${ }^{3}$, Nigel D. Browning $^{2}$, James E. Evans ${ }^{2}$, Nathan C. Gianneschi ${ }^{1}$.

${ }^{1}$ Department of Chemistry \& Biochemistry, University of California, San Diego, La Jolla, California 92093, United States

${ }^{2}$ Fundamental Computational Sciences Directorate and Environmental Molecular Sciences Laboratory, Pacific Northwest National Laboratory, Richland, Washington 99354, United States

${ }^{3}$ Department of Industrial and Manufacturing Engineering, Florida State University, Tallahassee, Florida 32306, United States

Transmission Electron Microscopy (TEM) and Scanning TEM (STEM) are extremely powerful techniques for characterizing nanomaterials in solution due to the high spatial resolution, the ability to determine internal and 3D structures and the possibility of obtaining elemental information through the use of energy dispersive x-ray spectroscopy (EDS) and electron energy loss spectroscopy (EELS). Due to the high vacuum requirement of the microscope column, nanoparticles in solution are typically imaged through one of two techniques a) dehydration onto a TEM grid (dry state TEM) or b) vitrification of thin films of solution on a TEM grid (cryo-TEM).

While both these techniques allow particle size and morphology to be readily determined, the process of dehydration or vitrification removes the possibility of studying particle dynamics. Recent developments in microchip fabrication have allowed for the commercialization of vacuum tight flow cells with 'electron transparent' silicon nitride windows. This technology permits TEM of nanoparticles in liquids at ambient temperatures and pressures and has been used to observe inorganic nanoparticle motion and growth. [1]

Metal-Organic Frameworks (MOFs) are crystalline materials which sit at the interface between organic and inorganic chemistry. Their extremely high internal surface areas $\left(>2,000 \mathrm{~m}^{2} / \mathrm{g}\right)$, as well as ease of structure modification through variations in both metal and ligand, make them extremely attractive materials for gas storage and separation. [2]

Currently there is no literature on the TEM of MOFs using liquid flow cells, however, with this technique it should be possible to observe for the first time individual particle formation and changes to morphology, which would provide a unique insight into their dynamics and potentially result in a better understanding of MOF performance. However, in-situ liquid TEM of nanoparticles presents many challenges, as particle motion is vastly restricted within the cell due to near wall effects [3] and e-beam damage to the particles and the solution can result in a variety of artifacts. [4]

In this paper we show that MOFs such as UiO-66 (Figure 1) and ZIF-8 can be imaged in liquids (Figure 2 ), we investigate damage mechanisms under different imaging conditions (Figure 3 ) and discuss the possibility of observing MOF formation in-situ. Prior to any quantitative analysis of these systems, the optimal conditions, free of artifacts must be found. While beam damage is a crucial problem for controlling experiments in the fluid stage for any system [3], [4], it is even more important to organic materials. Figure 1 shows bright field STEM images of UiO-66 in liquid water at $10 \mathrm{mg} / \mathrm{mL}$. The images show both a high number of particles within the window and that they can be imaged at high 
magnification. Initial experiments in TEM and STEM mode at $300 \mathrm{kV}$ show primary knock-on damage results in a decrease in particle size over time (Figure 3 left and center). However, reducing the voltage to $80 \mathrm{kV}$, results in particle size increase due to agglomeration of materials onto the particles (Figure 2 right), probably as a result of particle charging or damage due to the production of aqueous electrons and subsequent reactions.

After assessing damage mechanisms particle formation is investigated within the liquid cell and compared to particle formation in the bulk. Particle formation takes place in methanol, which provide additional challenges due to its high volatility and optimal loading conditions need to be established.

\section{References:}

[1] J. N. de and F. M. Ross, Nat. Nanotechnol., 2011, 6, 695-704

[2] H.-C. Zhou, J. R. Long and O. M. Yaghi, Chem. Rev., 2012, 112, 673-674

[3] White et al., Langmuir, 28, 3695, (2012)]

[4] T. J. Woehl, et al., Ultramicroscopy, 2013, 127, 53-63

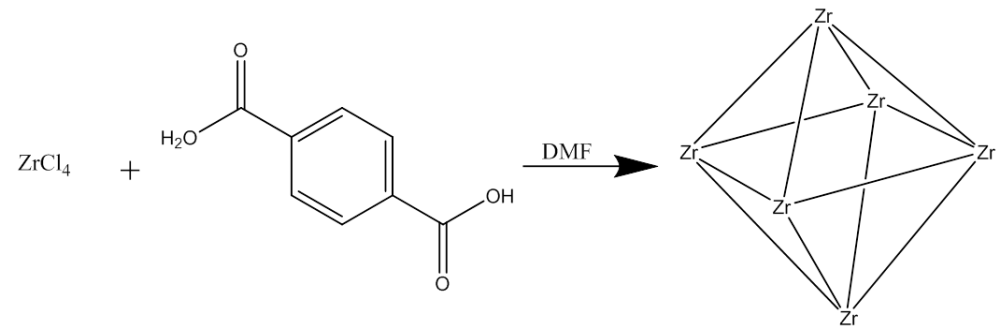

Figure 1. Schematic of UiO-66 synthesis.
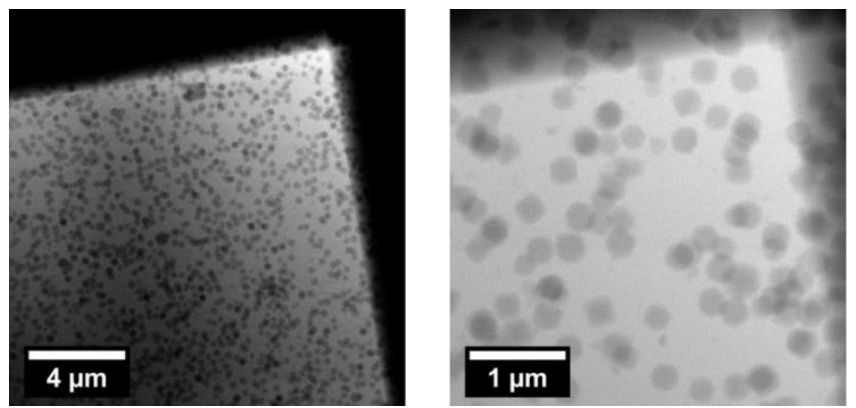

Figure 2. STEM images of UiO-66 in water at $10 \mathrm{mg} / \mathrm{mL}$ at $300 \mathrm{kV}$
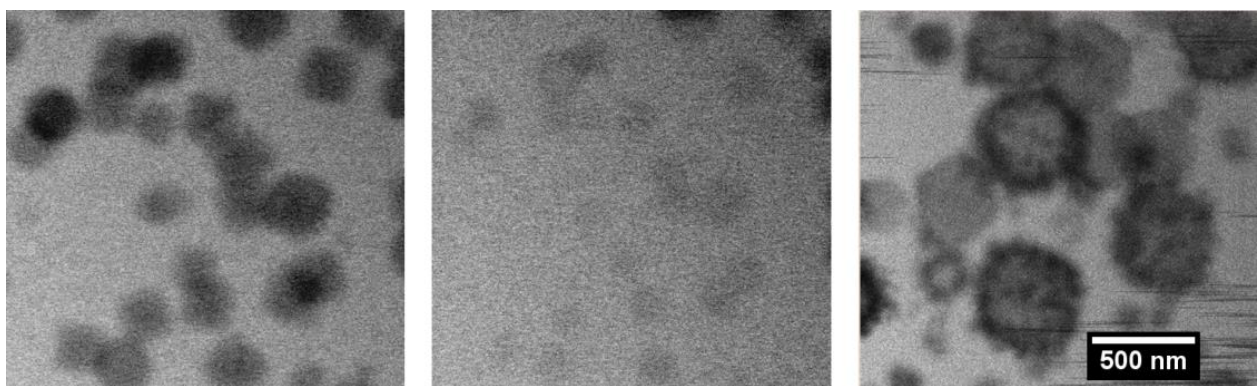

Figure 3. STEM images for UiO-66 in water at $10 \mathrm{mg} / \mathrm{mL}$, before damage (left) after damage at $300 \mathrm{kV}$ (center) and after damage at $80 \mathrm{kV}$ (right). 